\title{
Expansions on a computerized operant laboratory: Considerations for combining teaching and research
}

\author{
DAVID M. SARGENT \\ State University of New York at Oswego, Oswego, New York \\ and \\ THOMAS DEELMAN \\ State University of New York at Binghamton, Binghamton, New York
}

\begin{abstract}
The present laboratory configuration addresses concerns beyond the functional workings of a computerized research system to include human factors. The logistical aspects of error-free multioperator operations are discussed along with the economics of developing, assembling, and maintaining the stations. The actual configurations (hardware and software) of the present laboratory are explained and discussed, as are the philosophies and experiences that led to a highly successful combined research and teaching operant laboratory.
\end{abstract}

The primary purpose behind the development of the laboratory described in this paper was to assemble a system versatile enough to run a wide variety of operant conditioning paradigms, maximizing for ease of operation. In addition to the automation required to allow for flexibility in experimentation, human factors principles were utilized to facilitate the use of the lab by many different researchers, including various student assistants. The paradox of automation is that the more systematic the process, the less the involvement of the experimenters. The result is a greater potential for unnoticed problems and more opportunity for mistakes to be made due to automation-induced laxness. Finally, the less involved the student experimenter is with the process, the less he or she learns.

Anyone who has ever taught an experimental laboratory course in operant conditioning knows that it is often difficult to decide how much to have the students do "by hand." A good example would be the development of basic schedules of reinforcement. The slow emergence of the fixed-interval scallop is nearly impossible to portray enthusiastically in a lecture, and then does not easily become clear if the student in the laboratory merely puts the animal in the box, turns on the computer, and removes the animal after a session. The answer does not lie in returning to the time before the technology of today, but rather in a blend of today's technologies that optimizes error reduction and experimenter involvement. In the present laboratory, student researchers record the data by hand from the computer screen at set intervals. This on-screen data can be portrayed numerically, graphically,

Correspondence should be directed to D. M. Sargent, Department of Psychology, State University of New York, Oswego, NY 13126. or even in the classic cumulative chart format to facilitate experimental involvement.

\section{Setup Specifications}

To optimize flexibility while maintaining cost effectiveness without the use of a special dedicated controller (see, e.g., Palya \& Walter, 1993), four computers control a total of 12 operant chambers (3 chambers per computer). Two $28-\mathrm{V}$ dc electromechanical power supplies divide the laboratory into two electrically isolated halves. Previous arrangements did not have the capability of concurrent, dissimilar operations. It is often necessary, in our laboratory, to run 6 subjects on an experimental task while shaping another 6 on a different task. The present laboratory capability of staggering groups of subjects, whether across paradigms or even starting times, significantly increases the functionality of the laboratory. Centralized power distribution panels, one for each set of six chambers (see Figure 1), allow for two independent experimental sessions to run simultaneously.

With all power switches (for monitors, computers, interface, fans, and electromechanical equipment) in a single location, experimenter errors (e.g., forgetting the interface power) are eliminated. Although some problems may be eliminated simply by ganging components together on a single switch, items such as chamber fans, which are necessary for experimental sessions but irritating during program development and testing, are put on individual switches for each set of three chambers, with prominent indicator lights. The addition and orientation of visual indicators has been shown to enhance signal detection (Green \& Swets, 1966). This is especially important across experimental replications, where efficiency may be degraded by the repetitious nature of the task (Naitoh \& Townsend, 1970). This combination of au- 


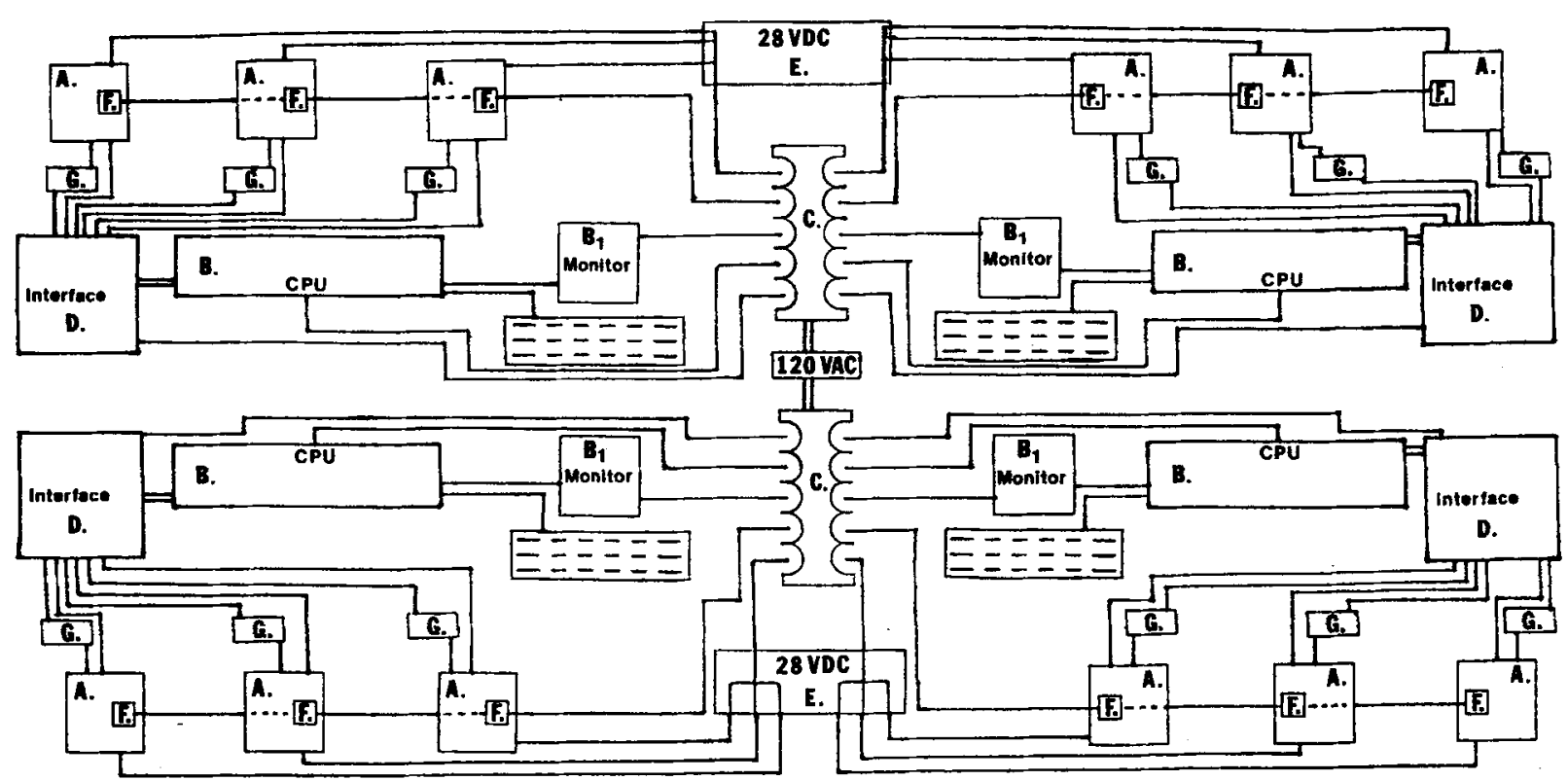

Figure 1. Schematic layout of the present laboratory with 12 operant chambers (A) controlled by four microcomputers (B and B1). Power controls (C) are centrally located and separate for each microcomputer and interface (D) as well as 28-V de power distribution (E) and chamber fans (F). Individual barpress debounce/one-shot circuits (G) are connected to each chamber.

ditory and visual cues eliminates the possibility of sessions' being run without fans, a significant confounding variable often experienced in previous laboratory setups. The conditions of signal detectability as a function of background noise have been shown to be related to signal type and modality (Fidell, 1974). Additionally, the top-down ordering of the power distribution switches is instrumental in eliminating problems due to different experimenters' turning things on or off in different orders. With the present system, power to the electromechanical food pellet dispensers (stepper motors operated by a specified pulse) does not occur until the computer has had a chance to clear the outputs from the interface, thereby eliminating overpulsing and the burning out of stepper motors in the dispensers (a costly problem).

\section{Types of Paradigms/Programs Currently Used}

As of this writing, the paradigms being used include a multiple fixed-ratio (MFR) program, a progressive ratio (PR) program, and a differential reinforcement of low rates of responding (DRL) program. These programs currently use schedule criteria for session completion rather than fixed time parameters. The MFR program and the DRL program require the subject to earn a set number of reinforcements to complete the session. The PR program increases the ratio of barpresses to reinforcement to an unlimited ceiling, with the session terminating only when the subject has not responded for a full $2 \mathrm{~min}$. In addition to the initial requirements of the paradigm, the DRL, MFR, and PR programs have flexible individual start times. Specifically, as each animal is put into the chamber and the sound enclosure is closed, the paradigm can start in direct response to the animal's behavior. This greatly re- duces order effects, primarily in the initial lag times in the ratios. In addition, if different student assistants are putting the animals in the chambers and, of course, are operating in slightly different sequences, these primary lag times do not develop into major experimental confounds.

The above programs have variable parameters only where current study variables are involved. The MFR program allows for two sequential ratios to be implemented, preceded by a short set of continuous reinforcements (CRFs). The number of CRFs is queried by the computer, as are the fixed ratio parameter and duration (number of reinforcements earned) of each of the sequential ratios. In the PR program, just the CRF and the progression increment number are queried, because the duration is determined by subject endurance. The DRL program queries the amount of time required for the subject to withhold responding and the number of total earned reinforcements to complete a session. There are no initial CRFs in the DRL session. These specific inputs require the experimenter to confirm the relevant experimental parameters while still preserving the efficiency of the computer interaction (Williges, 1984). The limited number of variable parameters required for each session is a good example of specific automation's greatly reducing the possibility of errors and the overall time required of the experimenter without necessarily limiting the apparatus in any hard-wired way. The primary point here is that we are maximizing for reduced experimenter error and flexibility simultaneously, as opposed to the usual compromise of automated error control at the expense of flexibility.

Flexibility has been found to be greatly facilitated through the utilization of a variety of programming lan- 
guages. Primary use has been through versions of BASIC (GWBASIC, ABASIC, QBASIC, ZBASIC, Visual BASIC, TurboBASIC, and full featured QuickBASIC). Additional advantages are gained in the implementation of modular languages, such as FORTH. Many researchers have "pet" languages that can be easily adapted to the existing interface system, including C, Pascal, Fortran, and even assembly language. With the majority of languages, access to the interface device is a simple case of reading inputs and writing outputs. Nearly all versions of BASIC utilize the program words OUT for writing outputs and IN for reading an input. For some high-level languages, access to the interface requires some type of CALL routine, and, on rare occasions, machine or assembly code must be compiled to create input and output commands. Our laboratory recently incorporated a public-domain version of FORTH for use both as a research and a teaching tool. The modular aspect of FORTH allows us to use the commands P@ (read specified input port) and P! (write specified output port) to define commands like FEED which would access the correct port address and send the correct pulse length to the device. This defined word is then placed in a library, making programming much simpler and more intuitive for both the student and researcher. All of the possible software approaches can be used without changing any wiring or hardware configurations.

\section{Present Laboratory Configuration}

Three primary aspects contribute to the success of the present laboratory. The first is the mixture of old technology and new technology, resulting in overall cost reductions. That is, most of the old relays and electromechanical equipment are readily available, if not already present in the lab, making the apparatus setup more familiar to people who have used the old equipment and less foreign to newcomers who understand basic relays, stepper motors for feeders, and standard $28-\mathrm{V}$ dc systems. The older $28-\mathrm{V}$ dc electromechanical equipment, such as that produced by the Ralph Gerbrands Company (Arlington, MA), the Lafayette Instrument Company (Lafayette, IN), and Coulbourn Instruments, Inc. (Lehigh Valley, PA), readily interfaces with any computer system through a variety of third-party interface devices (e.g., the Alpha Bus system). Second, a careful mixture of auditory and visual signals based on human factors principles is used to reduce experimenter error and facilitate experimenter involvement. The third aspect entails a maximized level of flexibility, primarily through software and programming languages and secondarily through limited hardware adaptations. For example, the use of screw terminal blocks inside each chamber allows for easy inclusion of different manipulanda (nose poke rather than barpress) or event cue apparati (e.g., lights or tone generators).

Flexibility is often compromised when budget restrictions are applied. Optimally, the most flexible system would have an independent computer for each conditioning chamber, allowing different start times, easy re- sets and changes for individual subjects, and less overall impact from a system failure. This approach, while being the most expensive, also requires excessive repetition on the part of the experimenter. We have found in our laboratory that the fewer the steps required of the experimenter, even repetitive steps, the fewer the errors. The opposite approach, involving a single computer networked to a large number of chambers, not only reduces flexibility but dramatically increases the programming complexities. Although these complexities can be overcome in a laboratory dedicated to a single research team, they become counterproductive when multiple researchers using different languages and approaches utilize a single facility. The compromise reached in our laboratory has a single microcomputer controlling three conditioning chambers simultaneously. Programming easily handles the possible problems of staggered starting times for the three chambers by way of independent timers or program flags on a real-time clock.

\section{Specific Hardware Configuration}

Real-time computer control of external events has always entertained the problem of switch debouncing. In addition to the fact that many of the mechanical switches used in an operant conditioning setup have extremely sloppy electrical properties, switches such as the barpress do not provide a uniform discrete electrical event. Programming can handle the situation where the onset of a barpress increments a variable once but not again unless the barpress was offset (some of our rats have been known to lean on the bar for a full minute!). However, this idea of using the CPU as a pulse former is quite inefficient. Pulse forming (electromechanically speaking) or one shot (electronically speaking) is best kept out of the computer, especially if there are several switches for which a single CPU has to keep track. Dedicating CPU clock cycles to the task of debounce delay can lead to other programming bottlenecks. The present system utilizes dedicated pulse formers to send switch closures to a digital input interface debounced with a series of individual debouncing circuits, as seen in Figure 2.

The issue of sending the correct pulse size to the feeder can also be handled as a lower level system. In fact, the system can be wired to allow the same pulse former to operate the feeder (this is feasible only in paradigms where reinforcement is contingent directly and immediately on a barpress response).

As mentioned earlier, automation can go so far as to leave the experimenter completely out of the data-collection loop. Some involvement in the data collection is necessary to keep the experimenter actively involved and to enable monitoring for possible problems and system faults. The flexibility of the computer screen allows anything from emulation of electromechanical dials and readouts to cumulative graphical displays of the data as the session progresses. For complex data collection, the choices range from color coding the data on the monitor to automatically saving all data to disk and backing it up to a secondary disk. 


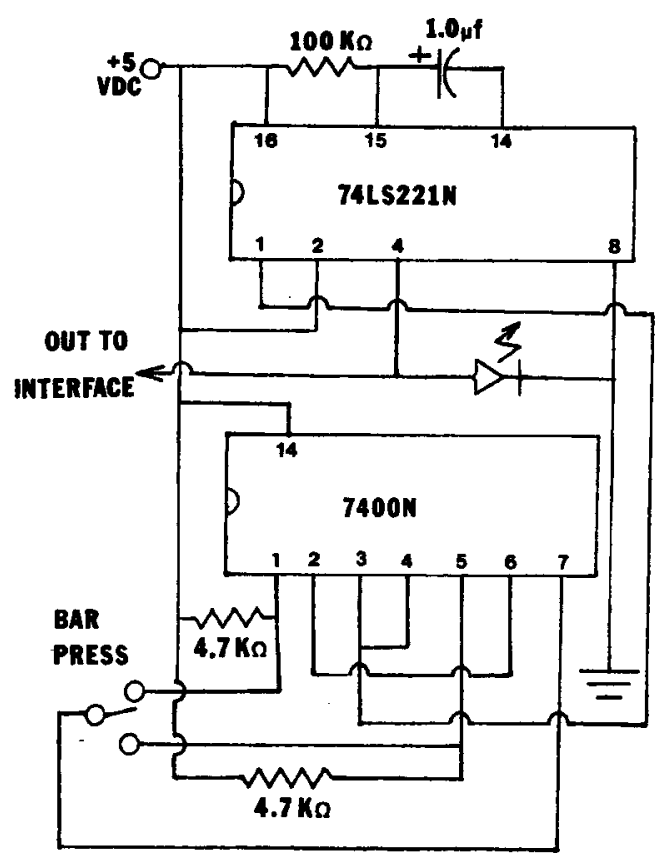

Figure 2. Switch debouncer with gated one-shot is shown for a single barpress manipulandum. Both $74 \mathrm{LS} 221 \mathrm{~N}$ and $7400 \mathrm{~N}$ can be wired for dual debouncer/one-shot circuit operation.

There are many choices for interface hardware. Many of the suppliers of other animal-care equipment (such as Coulbourne) also have interface hardware available. As can be expected, the complexity of the system is in direct relation to the price of the hardware, but for most operant conditioning paradigms, the most basic digital interfaces will suffice. The simple systems are usually capable of recording millisecond interresponse intervals across eight inputs simultaneously. Output control is usually handled with either relay or stepper control, both digitally modulated. Analog conversions, if needed for things like temperature data, have also become quite affordable and most interface systems have add in (on) A/D converters.

Recent laboratory upgrades have included the replacement of the obsolete computers (Sargent, 1991) with $8048633-\mathrm{MHz}$ systems from Gateway 2000 (North Sioux City, South Dakota). The prime advantage of these systems is the AnyKey keyboard with keyboard macro capability. Essentially the keyboard macro allows the use of a single, multikey command to sequentially implement a series of commands from either inside or outside a programming environment. An example from our laboratory uses a single key to call one program to clear the interface, clear TPA, load the necessary program for the current experimental run, and start it. Obviously this type of one-, two-, or three-key command has many advantages over poster lists of "startup procedures" for each student researcher to hopefully follow religiously in each and every experimental session. Again, a human factors approach allows for an easily remembered twoor three-key sequence that cannot be easily pressed by accident or mistaken for some other operation. A prime example would be the development of distinctly different sequences for saving accumulated data to disk and clearing the computer systems prior to an experimental session.

\section{Economic Flexibility}

Cost effectiveness was preserved in the present laboratory by using 80386 machines and not the latest, highspeed processor. The difference between 33-MHz 80386 and $66-\mathrm{MHz} 80486$ or Pentium is lost on a system using 8-bit interfaces to record response times in milliseconds. If, however, experiment programming requires memoryintensive environments such as Windows, larger data busses and faster clock speeds may be desired. Similarly, if the behavioral data involve subject movement across three axes or video analysis, a large fast processor would be required. The choice of equipment lies primarily with the interface device. An interface that is compatible across many different computers and is not dedicated to any specific input or output device is ultimately more flexible for varied experimentation, computer upgrades, and even laboratory expansions. This type of flexibility comes at the cost of extra time required to set up the systems compared with that required by the prefabricated systems from behavioral research equipment companies.

\section{REFERENCES}

Fidell, S. (1974). Prediction of aural detectability of noise signals. Human Factors, 16, 373-383.

GREen, D., \& Swets, J. (1966). Signal detection theory and psychophysics. New York: Wiley.

NAITOH, P., \& ToWNSEND, R. (1970). The role of sleep deprivation research in human factors. Human Factors, 12, 575-585.

Palya, W. L., \& Walter, D. E. (1993). A powerful, inexpensive experiment controller or IBM PC interface and experiment control language. Behavior Research Methods, Instruments, \& Computers, 25, 127-136.

SARGENT, D. M. (1991). An economical system incorporating obsolete computers in an operant conditioning laboratory. Behavior Research Methods, Instruments, \& Computers, 23, 273-276.

WiLliges, R. (1984). The tide of computer technology. Human Factors, 26, 109-114.

(Manuscript received November 22, 1994; revision accepted for publication January 19, 1995.) 